Review Article

\title{
Vascular Cognitive Impairment through the Looking Glass of Transcranial Magnetic Stimulation
}

\author{
Giuseppe Lanza, ${ }^{1}$ Placido Bramanti, ${ }^{2}$ Mariagiovanna Cantone, ${ }^{1}$ Manuela Pennisi, ${ }^{3}$ \\ Giovanni Pennisi, ${ }^{4}$ and Rita Bella ${ }^{5}$ \\ ${ }^{1}$ Department of Neurology IC, I.R.C.C.S. "Oasi” Institute for Research on Mental Retardation and Brain Aging, \\ 73 Via Conte Ruggero, 94018 Troina, Italy \\ ${ }^{2}$ I.R.C.C.S. Centro Neurolesi "Bonino-Pulejo", Via Provinciale Palermo, Contrada Casazza, 98124 Messina, Italy \\ ${ }^{3}$ Spinal Unit, Emergency Hospital "Cannizzaro", 829 Via Messina, 95126 Catania, Italy \\ ${ }^{4}$ Department of Surgery and Medical-Surgical Specialties, University of Catania, 78 Via S. Sofia, 95123 Catania, Italy \\ ${ }^{5}$ Department of Medical and Surgical Sciences and Advanced Technology, Section of Neurosciences, University of Catania, \\ 78 Via S. Sofia, 95123 Catania, Italy
}

Correspondence should be addressed to Giuseppe Lanza; glanza@oasi.en.it

Received 18 November 2016; Revised 30 January 2017; Accepted 5 February 2017; Published 26 February 2017

Academic Editor: Raj Kalaria

Copyright ( 2017 Giuseppe Lanza et al. This is an open access article distributed under the Creative Commons Attribution License, which permits unrestricted use, distribution, and reproduction in any medium, provided the original work is properly cited.

\begin{abstract}
In the last years, there has been a significant growth in the literature exploiting transcranial magnetic stimulation (TMS) with the aim at gaining further insights into the electrophysiological and neurochemical basis underlying vascular cognitive impairment (VCI). Overall, TMS points at enhanced brain cortical excitability and synaptic plasticity in VCI, especially in patients with overt dementia, and neurophysiological changes seem to correlate with disease process and progress. These findings have been interpreted as part of a glutamate-mediated compensatory effect in response to vascular lesions. Although a single TMS parameter owns low specificity, a panel of measures can support the VCI diagnosis, predict progression, and possibly identify early markers of "brain at risk" for future dementia, thus making VCI a potentially preventable cause of both vascular and degenerative dementia in late life. Moreover, TMS can be also exploited to select and evaluate the responders to specific drugs, as well as to become an innovative rehabilitative tool in the attempt to restore impaired neural plasticity. The present review provides a perspective of the different TMS techniques by further understanding the cortical electrophysiology and the role of distinctive neurotransmission pathways and networks involved in the pathogenesis and pathophysiology of VCI and its subtypes.
\end{abstract}

\section{Background}

The modern concept of vascular cognitive impairment (VCI), which encompasses any degree of vascular-related cognitive decline [1], is deemed to be the most common cognitive disorder, with a growing impact on social and health care expenses [2]. Moreover, early onset of VCI is also highly frequent in older stroke survivors, as showed in different studies [3-5]. The VCI construct includes not only vascular dementia $(\mathrm{VaD})$, but also mixed dementia (vascular and degenerative) and vascular cognitive impairment-no dementia (VCI-ND), which refers to a subgroup of patients who manifest cognitive decline resulting from cerebrovascular injury but do not satisfy the diagnostic criteria of dementia $[1,2]$. In addition to cognitive impairment, mainly involving processing speed and executive functioning [6], VCI patients also show behavioral (i.e., apathy, irritability, psychomotor agitation, disinhibition, and aberrant motor behavior) and mood deficits (namely depression, with or without anxiety) that correlate with worsening of both cognitive and functional status [7]. Moreover, strokes of the basal ganglia and internal capsule increase significantly the risk of poststroke depression and executive dysfunction [8]. Dementia after stroke may encompass all types of cognitive disorders [9], 
whereas a state of cognitive dysfunction before the index stroke is termed "pre-stroke dementia," which may entail vascular changes as well as insidious neurodegenerative processes.

As known, white matter hyperintensities, commonly seen on brain T2-weighted magnetic resonance imaging (MRI), are associated with varying degrees of cognitive impairment in patients with stroke, cerebral small vessel disease, and dementia [10], although the pathophysiological mechanisms within the white matter accounting for cognitive dysfunction remain unclear. Nevertheless, the strong relationship between vascular white matter lesions (WMLs) and nonmotor sequelae has been established in large community-based populations [11-14], showing that cognitive and moodbehavior abnormalities may arise from the ischemic disruption of the prefrontal cortical-subcortical circuits [15]. Medial temporal lobe atrophy was also found to be a significant imaging predictor of early cognitive dysfunction in stroke survivors [16]. A novel association between irreversible astrocyte injury and disruption of gliovascular interactions at the blood-brain barrier in the frontal white matter and cognitive impairment in elderly poststroke survivors has been recently proposed. In particular, clasmatodendrosis was suggested as another pathological substrate linked to frontal white matter hyperintensities, which may contribute to poststroke or dementia due to small vessel disease [17].

However, cognitive dysfunction and functional limitations are also associated with depressive disorder in stroke survivors $[3,5,7,18]$. Even subcortical ischemic vascular disease, including silent lacunar infarcts and WMLs, may be associated with late-life depression, often referred as "vascular depression" [19]. In 1997, Alexopoulos et al. [20] named "depression-executive dysfunction syndrome of late life" a clinical picture characterized by psychomotor retardation, difficulties at work, apathy, lack of interest, and limited depressive ideation and insight, together with prominent executive dysfunction at neuropsychological tests (i.e., planning, working memory, and set-shifting).

Brain imaging widely support these findings and provide the neuroradiological correlate of VCI and vascular depression [21-23]. Patients with vascular depression associated to WMLs show distinctive clinical-psychopathological findings with respect to those with lacunar lesions $[13,19,24-$ $28]$, with different prognostic implications as well [21, 26, 29]. In particular, whereas depressive symptoms are similar between the two groups, executive dysfunction and deficit of information processing speed are more frequently reported in patients with WMLs than in those with lacunar state $[19,25,26]$. Moreover, depressed subjects with WMLs showed a more rapid decline of cognitive and motor performances, as well as the presence of gait abnormalities and urinary disturbances [24]. It is also noteworthy that the severity of subcortical WMLs, rather than lacunar state, is associated with development of depressive symptoms [28] and to a history of late-onset depression [13]; similarly, microstructural white matter abnormalities of frontostriatal-limbic networks are related to executive dysfunction and late-life depression [25]. Finally, different reports emphasize that WMLs and executive dysfunction are linked to both poor response to treatment and progression to chronic depression compared to those with lacunar infarcts $[21,26,29]$.

Although the understanding of different aspects concerning VCI grows over time, as reflected into a considerable body of literature published every year, VCI still remains a broad concept whose borders are not always well defined [30]. Probably, the most pressing challenge is the difficulty in discriminating the different forms of VCI and in recognizing the earliest stages from normal brain aging [31, 32]. Currently, the diagnosis of VCI, whether at the stage of VCI-ND or an overt picture of $\mathrm{VaD}$, is mostly based upon clinical and neuropsychological evaluation, together with structural and functional neuroimaging. The pathological diagnosis of suspected clinical $\mathrm{VaD}$ requires adequate postmortem brain sampling and rigorous assessment methods to identify the commonly observed substrates [33]. The need for screening and early diagnosis tools have focused the search to identify early biological and instrumental markers of disease process and progress. In this context, systematic neurophysiological assessment of VCI can aid the diagnosis and predict the response to drugs. Among neurophysiological techniques, transcranial magnetic stimulation (TMS) emerged as valuable noninvasive method for the functional evaluation of cerebral cortex and neurotransmission pathways involved in a number of psychiatric and neurological disorders, including cognitive impairment and dementia [34].

The present review provides a perspective of the different TMS techniques by further understanding the cortical electrophysiology and the role of distinctive neurotransmission pathways and networks involved in the pathogenesis and pathophysiology of VCI and its subtypes.

\section{Transcranial Magnetic Stimulation: Basic Principles and Application in Cognitive Neuroscience}

Clinically introduced approximately 30 years ago as a diagnostic tool to study the central motor pathways, today, TMS goes well beyond the simple assessment of the corticospinal tract. Indeed, it is able to provide novel insights into the pathophysiology of the neural circuitry underlying neurological and psychiatric diseases and to give in vivo information about the excitability of the human brain cortex and the conduction along corticospinal tract as well as the functional integrity of intracortical neuronal and callosal fibers [35-37]. TMS has also a strong talent to unveil and monitor motor system impairment in the preclinical phase of several neurological disorders [34] or systemic diseases with the central nervous system (CNS) involvement [38, 39]. Moreover, integrated approaches using neurophysiological techniques together with structural and functional imaging have allowed us to study the connectivity across motor and nonmotor areas [40-43]. Finally, by evaluating the effects of agonists or antagonists for specific neurotransmitters, TMS can selectively and noninvasively explore the function of glutamatergic, gamma-aminobutyric acid- (GABA-) ergic, monoaminergic, and cholinergic central circuits (the socalled "pharmaco-TMS") [44, 45]. 
TMS can be delivered to the same or different brain areas as single pulse, pairs of stimuli, paired cortical and peripheral stimulation, or as trains of repetitive stimuli at various frequencies.

2.1. Single-Pulse TMS. A single magnetic pulse applied to the primary motor cortex at adequate stimulator intensity elicits a motor evoked potential in contralateral target muscles, thus providing a functional assessment of the corticospinal conduction [46]. In particular, the latency of motor evoked potentials and the central motor conduction time, defined as the time interval difference between the motor evoked potentials induced by motor cortex stimulation and those evoked by motor root stimulation, are indexes of integrity of the corticospinal pathways. The motor evoked potential amplitude reflects an aggregate measure of the excitation state of output cells in the motor cortex, nerve roots, and the conduction along the peripheral motor pathway to the muscles [47].

The resting motor threshold is considered a global parameter of human brain excitability, as it is a compound measure of the membrane excitability of cortical motor neurons, neural inputs into pyramidal cells within the cortex, as well as the excitability of spinal motor neurons, neuromuscular junctions, and muscles [47, 48].

A suprathreshold TMS pulse applied to the primary motor cortex during a tonic voluntary contraction of contralateral muscles results in a suppression of the electromyographic (EMG) activity of those muscles lasting few hundred milliseconds [49]. This phenomenon, called contralateral cortical silent period, is exploited as a functional measure of intracortical inhibitory circuits [50, 51], mainly mediated by GABA-B transmission [52]. Conversely, the ipsilateral silent period, evoked by stimulating the muscle and hemisphere of the same side, is considered to reflect the interhemispheric corticocortical inhibitory mechanisms, and it is thought to be modulated by transcallosal output neurons projecting to contralateral GABA-ergic interneurons $[35,36]$.

2.2. Paired-Pulse TMS. The paired-pulse TMS paradigm [53] allows the assessment of measures of intracortical interneuronal functions and interactions, such as the short-latency intracortical inhibition and intracortical facilitation of the motor response $[53,54]$. Short-latency intracortical inhibition is probably mediated by the activity of intracortical GABA-A interneurons [55]; intracortical facilitation is more complex being probably related to the activation of a cortical circuit projecting upon corticospinal cells different from that preferentially activated by single-pulse TMS, and it seems dependent to a great extent on the activity of glutamatergic excitatory interneurons $[56,57]$.

2.3. Sensory-Motor Modulation and Plasticity. Using specific TMS techniques, it is possible to investigate the sensorymotor interaction within the cerebral cortex as well as the cortical phenomenon of the short-latency afferent inhibition and long-latency afferent inhibition. Short-latency afferent inhibition is thought to reflect the integrity of central cholinergic neural circuits, as it has been shown to be reduced or abolished by the muscarinic antagonist scopolamine in healthy subjects $[58,59]$ whereas it is positively modulated by acetylcholine [60]. It has been suggested that it may depend on the integrity of circuits linking sensory input and motor output [61], given that other neurotransmitters (such as dopamine) are supposed to play a modulatory role in the cholinergic transmission [62]. Long-latency afferent inhibition is probably related to the cortical-cortical connections involving the motor cortex and both primary and secondary somatosensory cortical areas [61].

TMS also allows us to study the synaptic plasticity phenomena at different levels. In healthy subjects, a magnetic stimulus applied after a brief period of exercise reveals phenomena of "post-exercise facilitation" and "delayed facilitation" that provide valuable information on cortical excitability and intracortical synaptic reorganization underlying motor learning [63].

2.4. Repetitive TMS. Single TMS pulses delivered in trains are the principle of repetitive TMS (rTMS), an approach that can transiently modulate the functioning of stimulated and connected brain areas mainly depending on the frequency of stimulation $[36,64,65]$. For this reason, rTMS might have therapeutic and rehabilitative applications since the effects of repeated sessions may persist in time [40, 48, 66-70]. The mechanisms of these changes are not completely clear but seem to be related to the phenomena of synaptic longterm potentiation (LTP) and long-term depression (LTD) within the CNS [71, 72]. Similarly, it is possible to induce LTP-like changes in the sensory-motor system by means of the paired associative stimulation [73], which induces a lasting increase of corticospinal excitability that can be considered as a marker of cortical plasticity $[73,74]$.

2.5. TMS in Dementia. Although the single neurophysiological abnormality revealed by TMS is not disease-specific [36], distinctive set of electrocortical changes that cosegregate specifically in different neurological and psychiatric disorders has been found, suggesting the involvement of specific biological substrates in their pathogenesis $[34,75,76]$. In the last years, a number of studies have shown abnormalities in TMS assays of cortical function in dementias, namely an increased excitability of the motor cortex in both Alzheimer's disease (AD) [77] and $\mathrm{VaD}$ [78].

Even if not always clinically evident, the involvement of motor areas in dementia has been demonstrated by clinical, neuropathological, and neuroimaging studies. Changes in motor areas may be secondary to the direct structural alterations caused by the disease process but, more often, they are the consequence of indirect remodeling mechanisms [34]. In this context, increasing evidences support the hypothesis that the phenomena of brain plasticity are involved in different kinds of dementia, related to functional and structural components, each entailing a number of cellular mechanisms operating at different time scales, synaptic loci, and developmental phases within an extremely complex framework [79]. However, the exact relationship between 
brain plasticity and excitability of cortical areas and their connections is not completely understood yet.

Table 1 summarizes all the TMS studies in patients with VCI and its subtypes [76, 80-97].

\section{TMS Correlates of VCI Subtypes}

3.1. TMS in Vascular Dementia. The majority of TMS studies indicate that the motor cortex of $\mathrm{VaD}$ patients is hyperexcitable (reduced resting motor threshold) [78], a common feature shared by $\mathrm{AD}$ [77]. This finding has been considered as part of a plastic compensatory mechanism in response to neuronal loss and/or ischemic injury [34, 40]. Accordingly, the enhanced excitability and plasticity might counteract cognitive decline and shed light on the reasons underlying decline or preservation of cognitive domains in dementing population $[87,88]$. This hypothesis has been demonstrated by means of TMS mapping study in AD patients, which showed a frontal and medial shift of the motor cortical output center of gravity, suggesting a functional reorganization of cortical brain areas, at least in the early stages [98]. A similar pattern has been also observed in subcortical ischemic $\mathrm{VaD}$ [84], which encompasses a homogeneous subtype of patients of particular interest because of the relatively slow progression, often making the differentiation from AD difficult $[1,2]$.

However, although a cortical reorganization similar to that occurring in AD was hypothesized also in VCI, it was not clearly demonstrated yet. Based on this assumption, Guerra and coworkers explored the relationship between excitability and plasticity in subcortical ischemic $\mathrm{VaD}$ [94]. Although obtained from a small sample size, they found that motor cortex had enhanced excitability in patients with $\mathrm{AD}$ and subcortical ischemic $\mathrm{VaD}$ with respect to controls. However, more interestingly, the motor cortex was plastically rearranged in both groups of patients, although with a slightly lesser center of gravity frontal shift in those with subcortical ischemic VaD compared to AD. Moreover, a significant direct correlation between parameters associated to cortical excitability and those associated to cortical plasticity was evident [94]. This would suggest the existence of mechanisms that partially overlap and probably act in the same neurophysiological way although they are, at least in principle, different both in location (subcortical versus cortical) and origin (vascular versus degenerative). The authors concluded that $\mathrm{AD}$ and subcortical ischemic $\mathrm{VaD}$ can share a common neurophysiological platform, related to the progressive neuronal loss within motor areas and to the ischemic disconnection, respectively [94]. This alteration could finally promote a functional rearrangement that allows the preservation of motor programming and execution despite disease progression $[90,94]$.

3.2. TMS in Vascular Cognitive Impairment-No Dementia. A crucial issue is whether it is possible to early identify VCI-ND subjects at risk for clinical progression. In a previous study on nondemented elderly patients with subcortical vascular disease and clinical-cognitive profile of VCI-ND [87], it was found that the ischemic interruption of prefrontal- subcortical loops implicated in executive functions and mood/affection regulation might result in functional changes of intracortical excitatory neuronal circuits, specifically an enhanced intracortical facilitation. Moreover, it has been shown that transcallosal inhibitory mechanisms were spared in patients with leukoaraiosis, a finding which differs from those with degenerative dementia (such as AD), in whom a functional callosal impairment is observed even in the preclinical or early stages [89].

A pilot TMS study after a 2-year follow-up has been performed on the same participants with VCI-ND [88]; compared to baseline, they showed an increase of global cortical excitability (reduction of the median resting motor threshold), along with a significant worsening of neuropsychological tests evaluating frontal lobe abilities, although without reaching a substantial functional impairment. We considered these findings to be part of the plastic compensatory mechanisms following the loss of motor cortical neurons, supporting the concept of VCI as a dynamic condition and some TMS changes as indexes of motor cortex plasticity [74]. Accordingly, it has been hypothesized that the critical point at which the resting motor threshold becomes abnormal might represent a "neurophysiological cut-off" to discriminate VCI patients advancing to $\mathrm{VaD}$ from those cognitively stable. If these patients show a "hyperfacilitation" at baseline, this would be considered as a trend of electrocortical dysfunction in "brain at risk" during the transition from normal brain aging to VCI, up to an overt $\mathrm{VaD}[30,88]$.

More recently, intriguing findings come from the investigation of central cholinergic circuit functioning by means of short-latency afferent inhibition. Unlike AD, the role of cholinergic involvement in $\mathrm{VaD}$ is indeed still under debate, and TMS data are limited and often conflicting [81, 82, 85]. In a recent paper aiming at the evaluation of short-latency afferent inhibition in a sample of VCI-ND patients with predominant WMLs, central cholinergic circuitry was found to be not clearly involved in patients compared to age-matched controls [95], suggesting a distinctive profile of the cholinergic pathway with respect to primary cholinergic forms of dementia (namely, AD), even in the early stage [99]. A reasonable explanation is that VCI may exhibit considerable interindividual variation in the location of subcortical infarcts and, therefore, in the distribution and magnitude of the resultant cholinergic denervation [100]. This study also supports the role of short-latency afferent inhibition in providing useful insight in the diagnosis and prognosis of different dementing process and in the identification of responders to acetylcholinesterase inhibitors [95].

3.3. TMS in Vascular Depression. Also at the TMS level, available data support the "vascular depression hypothesis" as a different syndrome with respect to nonvascular early-onset major depressive disorder. In particular, the depressive syndrome in vascular depression should be regarded as one of the clinical manifestations in the wide symptom spectrum of subcortical cerebrovascular disease rather than being a primary disease $[76,86]$. Consistent with this hypothesis, an increased intracortical facilitation was observed not only in nondepressed VCI patients but also in those with late-onset 


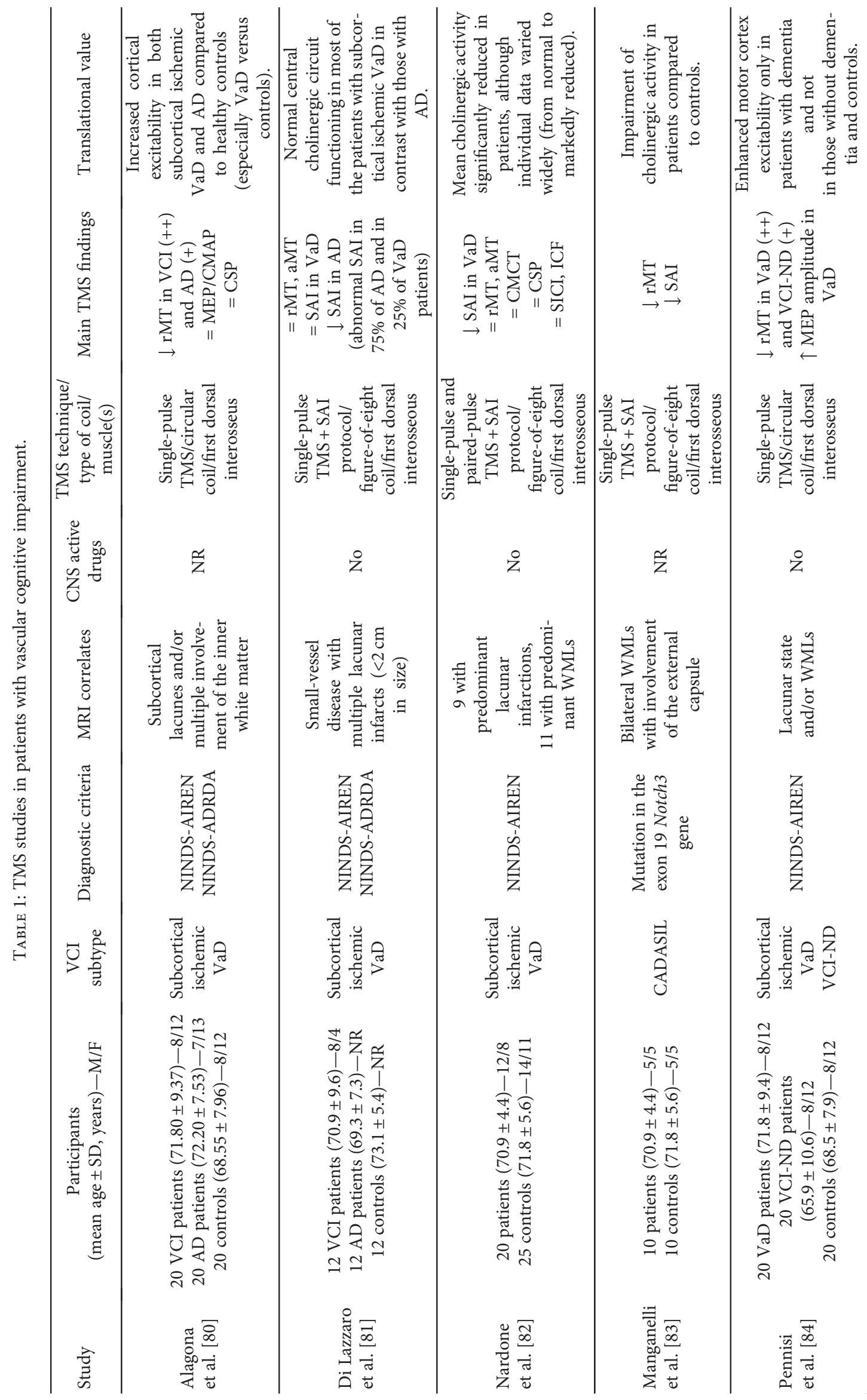




\begin{tabular}{|c|c|c|c|c|}
\hline 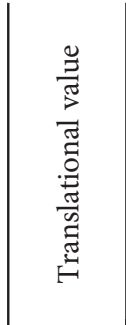 & 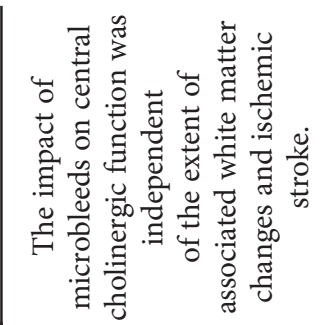 & 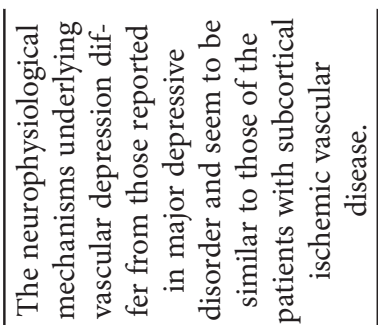 & 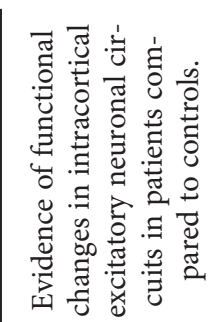 & 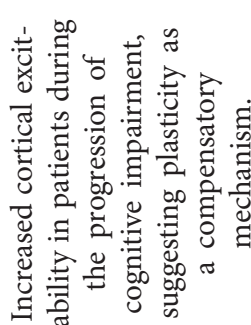 \\
\hline 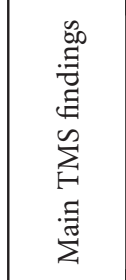 & 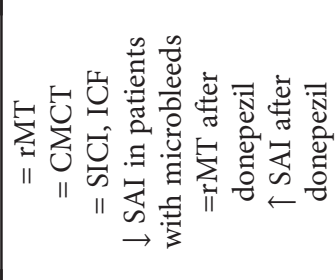 & 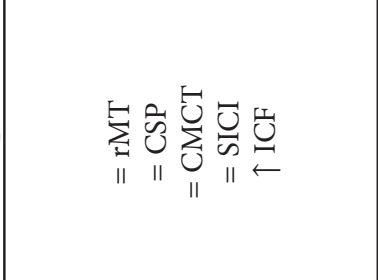 & 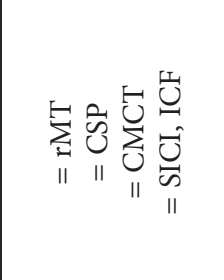 & 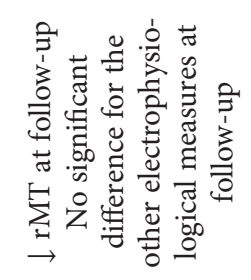 \\
\hline 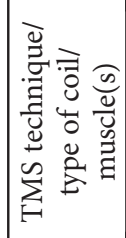 & 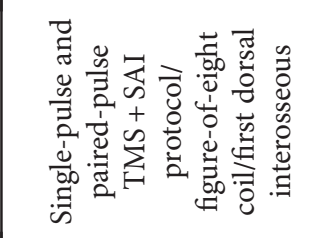 & 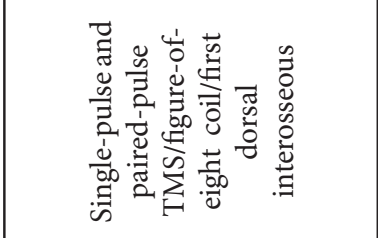 & 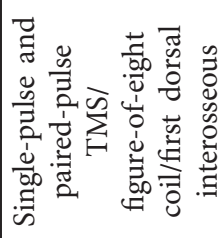 & 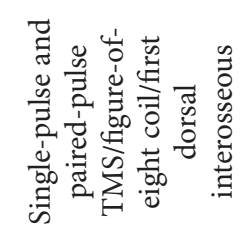 \\
\hline 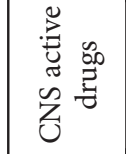 & 之o & $\stackrel{\circ}{z}$ & ¿ & $\stackrel{\circ}{z}$ \\
\hline 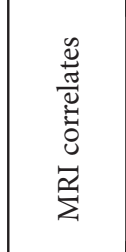 & 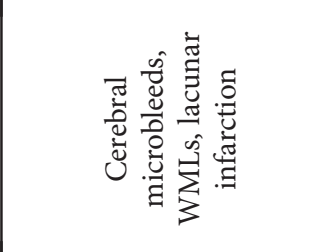 & 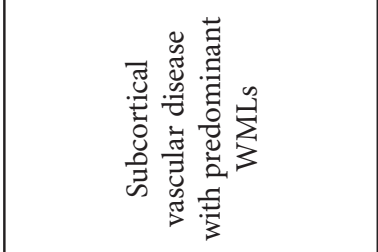 & 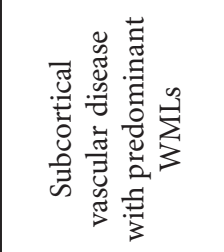 & 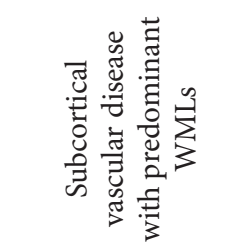 \\
\hline 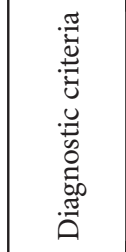 & 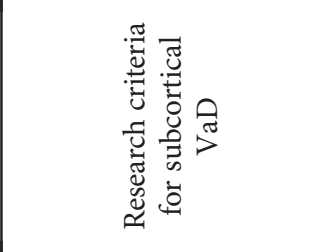 & 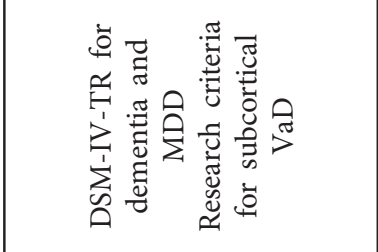 & 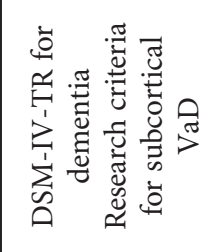 & 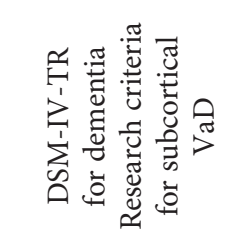 \\
\hline D. & 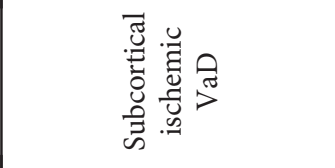 & $\begin{array}{l}\sum_{i} \\
\dot{d} \\
>\end{array}$ & 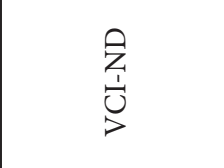 & $\begin{array}{l}\sum_{1} \\
\dot{0} \\
>\end{array}$ \\
\hline 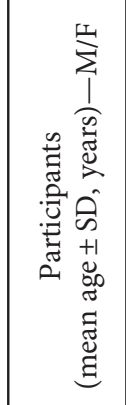 & 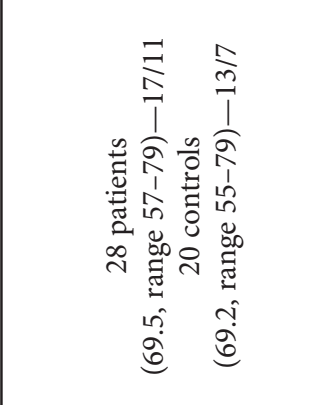 & 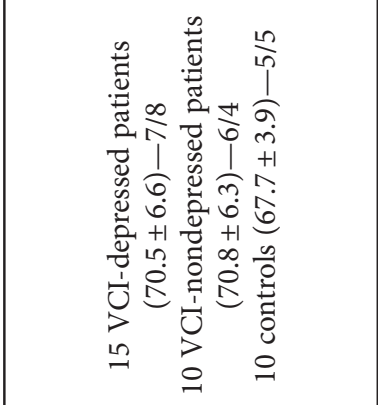 & 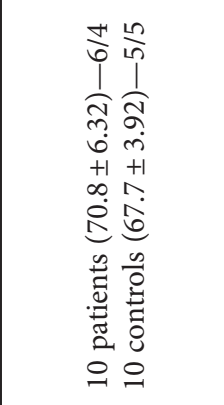 & 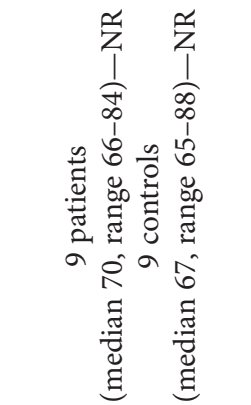 \\
\hline$\tilde{\omega}$ & 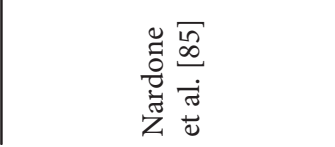 & 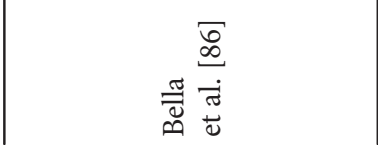 & 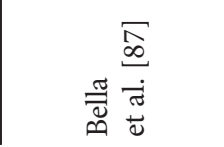 & 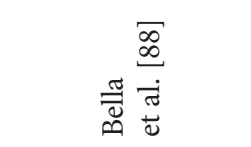 \\
\hline
\end{tabular}




\begin{tabular}{|c|c|c|c|c|}
\hline 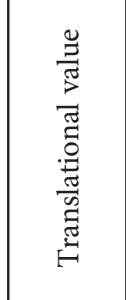 & 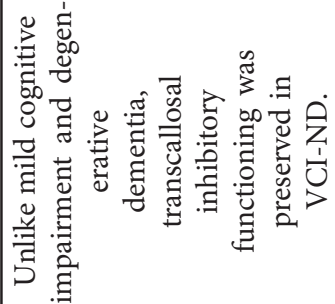 & 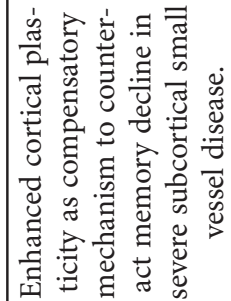 & 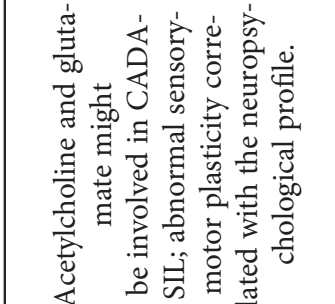 & 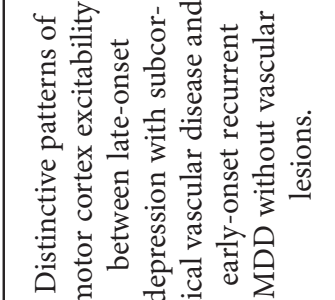 \\
\hline 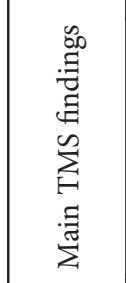 & 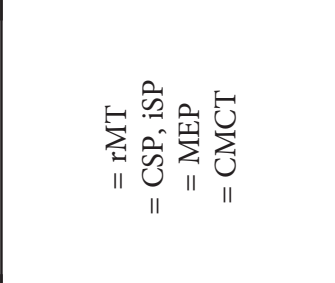 & 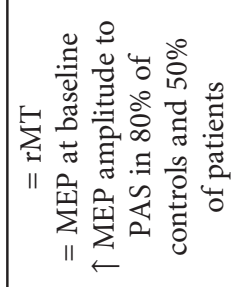 & 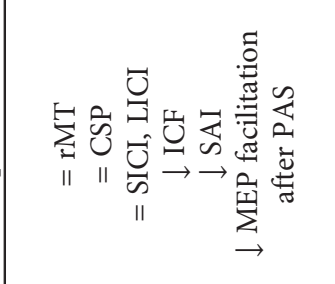 & 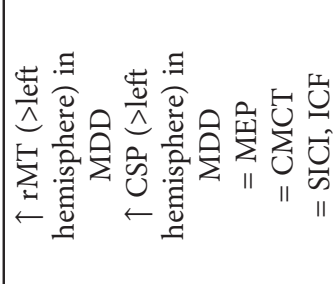 \\
\hline 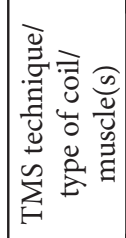 & 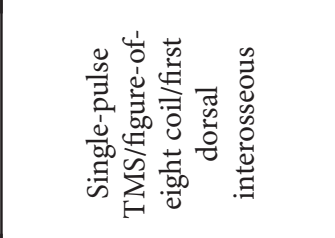 & 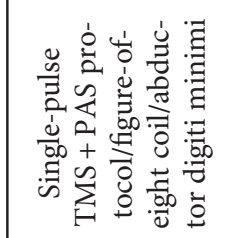 & 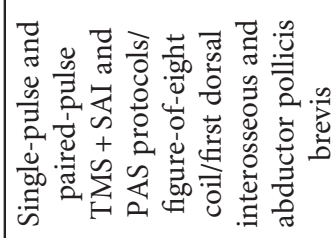 & 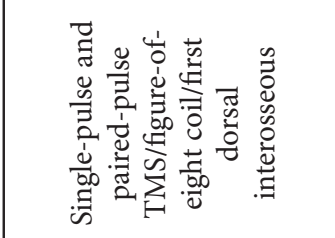 \\
\hline 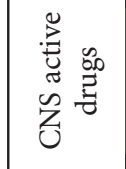 & $\dot{z}$ & ż & $\stackrel{\circ}{z}$ & 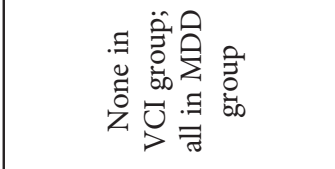 \\
\hline 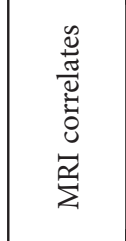 & 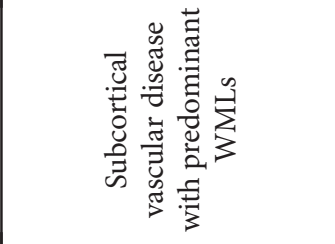 & 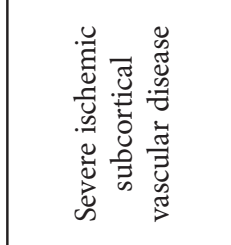 & 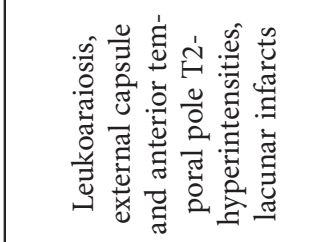 & 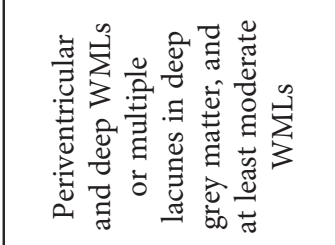 \\
\hline 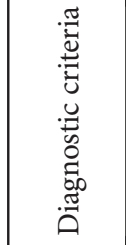 & 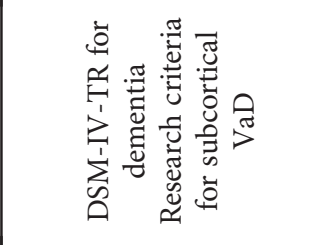 & 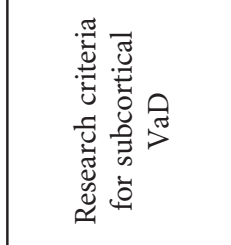 & 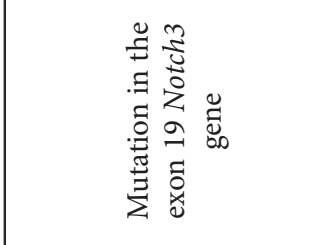 & 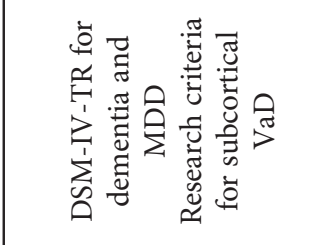 \\
\hline 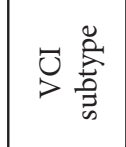 & $\begin{array}{l}\sum_{i} \\
\dot{i} \\
>\end{array}$ & 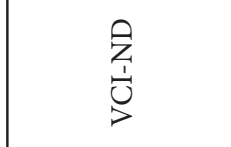 & 是 & $\begin{array}{l}\sum_{i} \\
\dot{j} \\
>\end{array}$ \\
\hline 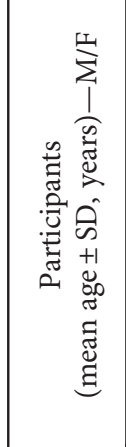 & 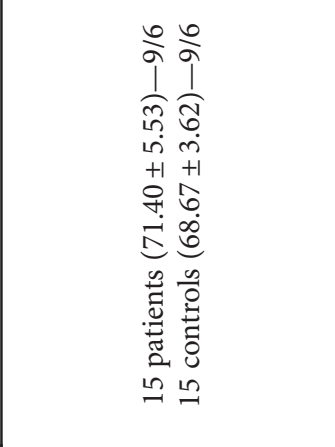 & 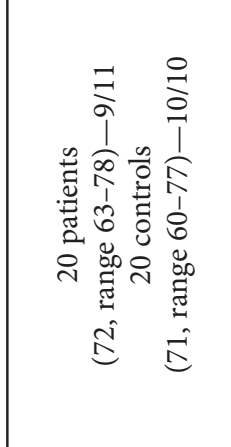 & 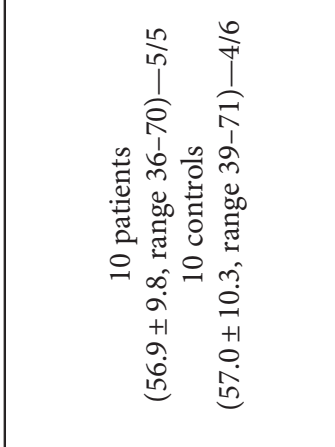 & 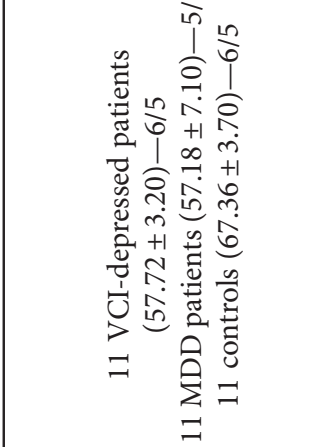 \\
\hline $\begin{array}{l}\vec{z} \\
\text { 离 }\end{array}$ & 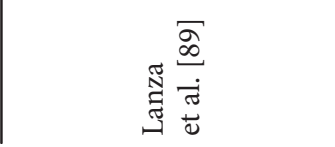 & 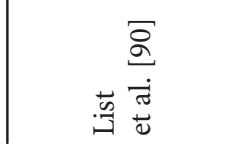 & 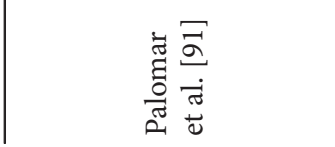 & 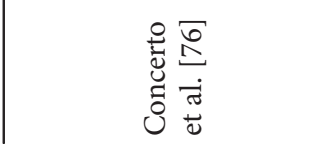 \\
\hline
\end{tabular}




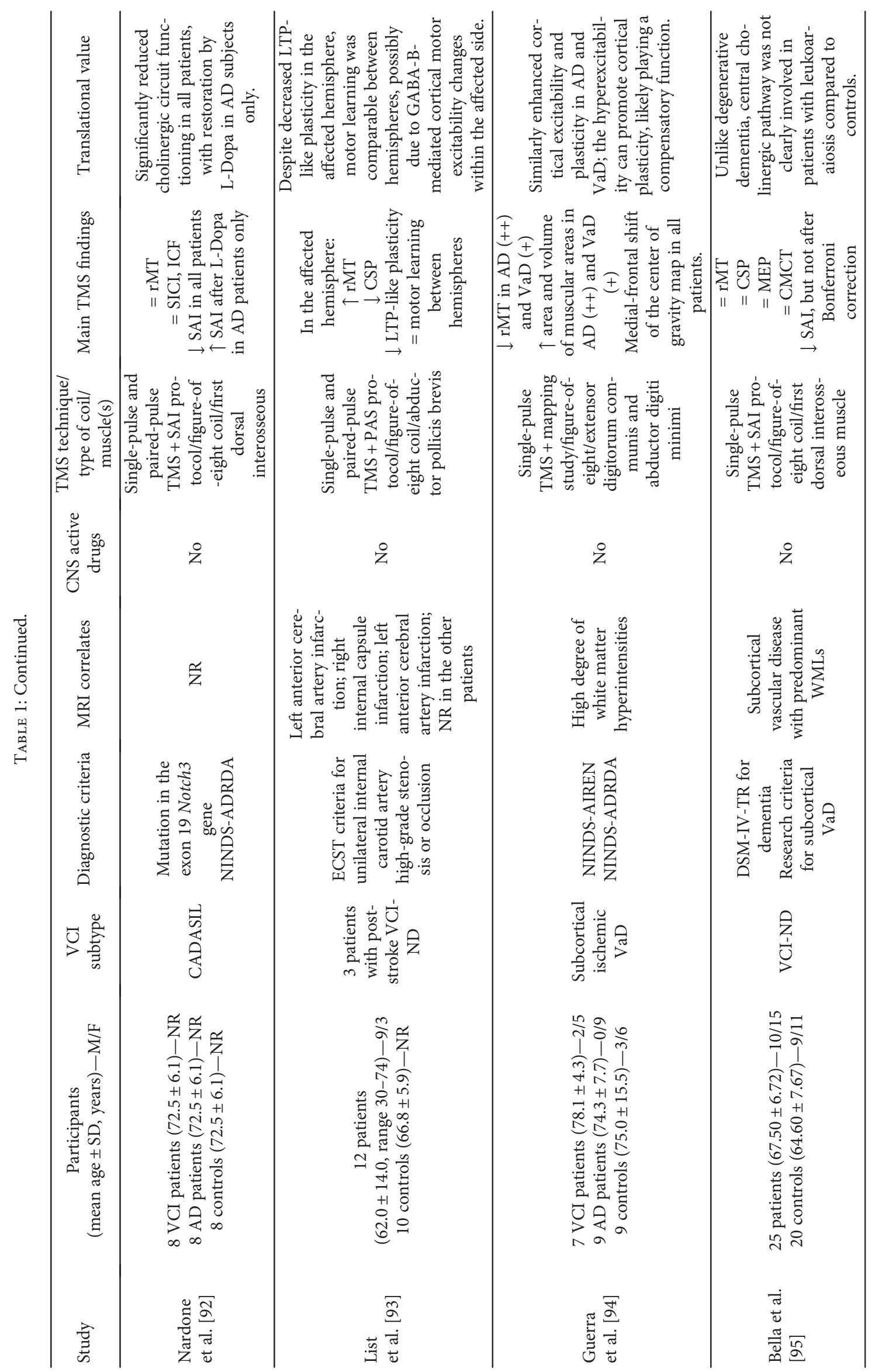




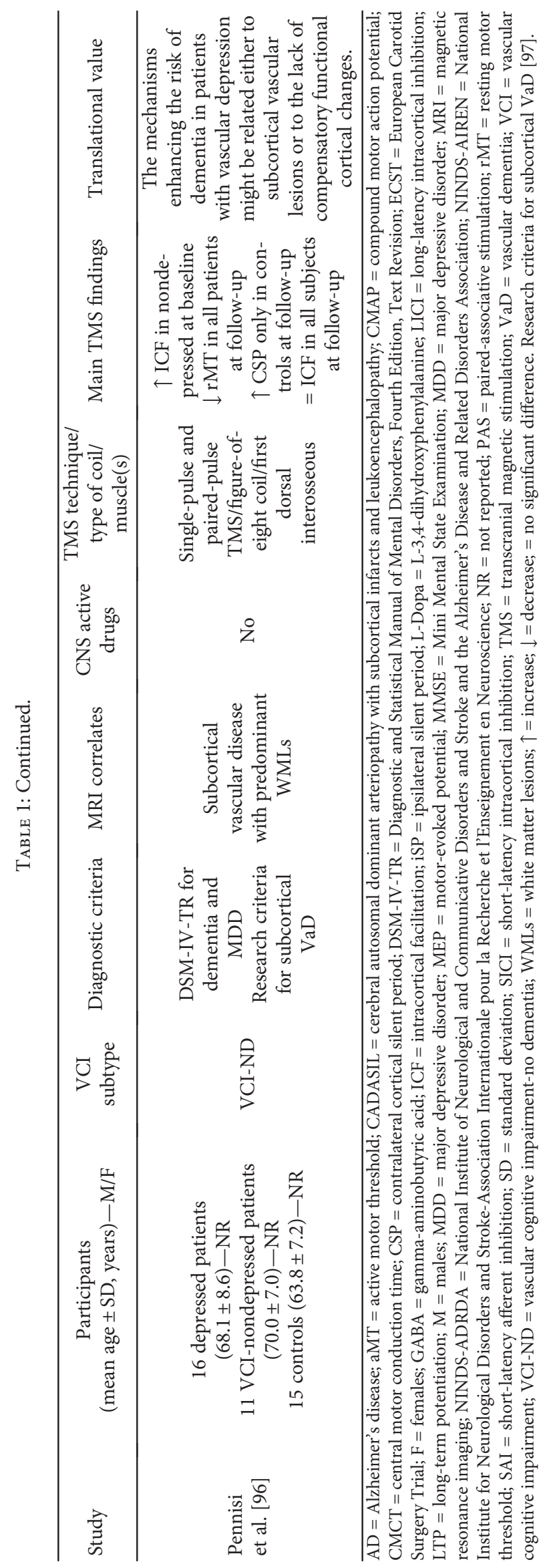


vascular depression [76, 86]; conversely, the level of intracortical facilitation in patients with early-onset major depression and without evidence of cerebrovascular disease was similar to healthy controls [76]. This result suggests that the disruption of frontal-striatal circuits by vascular lesions may predispose, precipitate, or perpetuate late-life depression. In particular, the vascular "disconnection" at the level of the dorsolateral prefrontal cortex (DLPFC) or the dorsal portion of the head of the caudate nucleus may affect the presentation and course of vascular depression [29].

However, at present, little is known about the impact of late-onset depression on plastic changes that could contribute to the preservation of cognitive functions in VCI patients. To better characterize the possible role of depression in cognitive decline of patients with vascular damage, we have recently investigated the relationship between the progression of the neurophysiological changes and cognitive impairment in patients with VCI-ND with those obtained in a group of patients with vascular depression and controls [96]. A high level of intracortical facilitation at baseline was found in nondepressed patients only, and it has been considered to be protective from cognitive decline, possibly through an enhancement of glutamaterelated neuroplasticity. At follow-up, a hyperexcitability was observed in both groups of patients, pointing out an involvement of glutamatergic neurotransmission as well, but without a specific neurophysiological change that parallels cognitive decline in depressed patients. This indicates that the mechanisms that contribute to cognitive deterioration in vascular depression might be related either to subcortical changes produced by vascular lesions or to the lack of compensatory functional cortical [96].

3.4. TMS in CADASIL. The cerebral autosomal dominant arteriopathy with subcortical infarcts and leukoencephalopathy (CADASIL) is due to mutations in the Notch 3 gene on chromosome 19 and causes progressive cognitive decline till dementia, cerebral ischemic events, psychiatric disorders, and migraine. It reasonably represents a pure genetic model of $\mathrm{VaD}$; these patients are therefore particularly suitable for exploring the relationship between ischemic brain injury, clinical manifestations, and TMS changes [78].

In 2008, Manganelli et al. [83] first demonstrated a dysfunction of motor cortex excitability and cholinergic innervations in terms of reduced resting motor threshold and shortlatency afferent inhibition. The recurrent vascular insults and the lesion burden in strategic areas typically involved in CADASIL [101], such as the external capsule [102], may lead to the interruption of corticosubcortical cholinergic circuits. Therefore, it has been hypothesized that the cortical hyperexcitability in CADASIL might be caused by cholinergic, GABA-ergic, and glutamatergic neuron dysfunction, supporting the hypothesis of several neurotransmitter system involvement in CADASIL, similar to other forms of dementia [78]. More recently, short-latency afferent inhibition was found to be significantly reduced in both $\mathrm{AD}$ and CADASIL patients, although administration of L-3,4-dihydroxyphenylalanine (L-Dopa) was able to significantly increase shortlatency afferent inhibition in the $\mathrm{AD}$ group only, suggesting that relationship between acetylcholine and dopamine systems may be specifically abnormal in $\mathrm{AD}$ [92].

The study of different intracortical circuits and sensorymotor plasticity using TMS protocols confirmed that acetylcholine and glutamate are involved in CADASIL and that abnormal plasticity correlates with the neuropsychological profile [91]. Similarly, cathodal transcranial direct current stimulation (tDCS) revealed no evidence of cortical dysplasticity in CADASIL, suggesting that increased rapid-onset cortical plasticity may contribute to largely preserve cognitive and motor functions despite extensive ischemic subcortical vascular disease [103].

\section{The Contribution of Neuromodulatory Techniques}

Repetitive TMS and tDCS are emerging as promising tools to modulate cortical circuits and related neurochemical pathways in dementing illnesses [104-106]. Several studies, although methodologically heterogeneous, have shown that specific paradigms of stimulation might improve cognitive performance and mood-behavioral symptoms, possibly becoming an alternative to conventional neuroleptic therapy for psychiatric symptoms of dementia [104]. Current pharmacological treatment, indeed, suffers from significant limitations, such as nonspecific mode of actions, an insufficient tailoring to the individual, and a number of adverse effects. The targets for an ideal nonpharmacological neuromodulatory treatment would be (a) modulation of activity in the targeted area, (b) modulation of activity in a dysfunctional network, (c) restoration of adaptive balance in a disrupted network, (d) guiding plasticity for best behavioral outcome, and (e) suppression of maladaptive changes for functional advantage.

In $\mathrm{AD}$, there is a general trend for improvements across a wide range of cognitive outcome measures following treatment with rTMS and tDCS, probably mediated by compensatory mechanisms supporting the residual abilities, even with long-lasting effects [104]. Typical sites of stimulation include the DLPFC, temporal regions, temporoparietal regions, or a combination of multiple regions. Interestingly, the benefits may be highly task-specific (i.e., action naming versus object naming and visual recognition versus spatial recognition), taking into account that dementia severity may affect the clinical response. Combining these techniques with cognitive rehabilitation might influence learning in a neuroplastic fashion [104].

In patients with vascular-related cognitive decline, rTMS data are very few. In a randomized controlled pilot study on patients with subcortical ischemic vascular disease and clinical picture of VCI-ND, Rektorova et al. [107] showed that high-frequency stimulation over the left DLPFC improved executive performance and hypothesized that long-lasting effects could be due to an indirect activation of monoaminergic neurons located in the midbrain (dopamine) and/or the brainstem (noradrenaline and serotonin) and their cortical and subcortical targets [107]. Among individuals with vascular depression, rTMS is mentioned as a nonpharmacological option, although WMLs load and global vascular risk profile 
are predictors of poor response [108]. Finally, a recent systematic review evaluating the effectiveness of rTMS in improving vascular depression and poststroke depression concluded that it was beneficial in treating depression among individuals with cerebrovascular disease over the short term. However, heterogeneous populations and variability in study design and protocol as well as a limited number of investigations challenge the ability to draw firm conclusions on the effectiveness of rTMS [109].

Very recently, exciting results come from preclinical studies showing the restorative effect of rTMS on cognitive ability in murine model of $\mathrm{VaD}$ and its impacts on hippocampal synaptic plasticity $[110,111]$. In this context, another possible mechanism of action of noninvasive brain stimulation in dementia is represented by the modulation of neurotrophin release. Indeed, experimental studies in rat models of $\mathrm{AD}$ have shown that tDCS can improve learning in mice through secretion of brain-derived neurotrophic factor (BDNF) and activation of tyrosine kinase B receptor [112]. Moreover, low-frequency rTMS might improve cognitive deficits through the upregulation of the hippocampal BDNF and the expression of the glutamate receptor for N-methylD-aspartate (NMDA) [113]. Finally, low-frequency rTMS in $\mathrm{VaD}$ model rats may improve learning and memory, protect pyramidal cells from apoptosis, and promote hippocampal synaptic plasticity through increased expression of the $B c l-2$ and reduced expression of Bax [114].

\section{Limitations, Critical Aspects, and Possible Solutions}

Although innovative, the approach based on noninvasive brain stimulation in vascular-associated cognitive impairment includes a number of potential criticisms:

(a) One is difficulties in recruitment of a sufficient number of age-matched controls without evidence of cerebrovascular disease at neuroimaging (that is strikingly prevalent among elderly) or with any cognitive impairment at neuropsychological evaluation.

(b) The correlation between different TMS patterns of cortical excitability and anatomical distribution and severity of vascular lesions has not been systematically investigated; therefore, without the contribution of advanced imaging, neuronavigated systems, or other electrophysiological techniques (i.e., highdensity EEG), the spatial resolution of TMS remains quite low. However, the majority of patients enrolled in the studies here reviewed exhibited predominant WMLs within the frontal lobes, thus partially limiting this variability.

(c) TMS-related measures of cortical excitability do not provide specific clinical information but are sensitive to the "global weight" of several neurotransmitters, as well as to subcortical and cortical motor inputs $[40,57]$. As a consequence, the description of TMS findings observed in the different VCI subtypes cannot be linked to behavioral changes. It is worth to highlight that the available TMS evidences in vascular-related cognitive disorders point out the possibility to identify a profile of cortical excitability related to each VCI subtype rather than a specific behavioral deficit. On the other hand, behavioral changes are common in all VCI patients, and therefore, the identification of a distinctive TMS correlate is challenging. On the contrary, studies in vascular depression allowed us to identify a more specific pattern of cortical excitability, thus suggesting the role of some TMS measures as putative markers of disease process and progress compared to nondepressed subjects.

(d) The available results on relatively small sample size might not be confirmed on larger populations, although most of them have been obtained from homogeneous samples in terms of demographics, clinical and neuroradiological features, and age matching with healthy controls.

(e) The hypothesis to identify a characteristic signature in patients with subcortical vascular disease at risk for developing $\mathrm{VaD}$ or mixed dementia could be risky given the paucity of previous data and the difficulty of similar approaches in other dementing conditions, such as non-AD dementias; consequently, the identification of a pattern of cortical excitability rather than single marker of the disease process and progress would be more reliable and reproducible [34].

(f) As known, vascular lesions, even in the absence of any motor deficit, give a significant contribution to the development and progression of degenerative dementia, so that it cannot be excluded that some patients enrolled in the studies here reviewed had a mixed form of dementia rather than a pure $\mathrm{VaD}$. In this respect, TMS is not currently able to clearly distinguish $\mathrm{VaD}$ from $\mathrm{AD}$ based only on their neurophysiological profile or to clearly disentangle the vascular from degenerative burden [30].

(g) Elderly population usually takes a number of drugs for the treatment/control of vascular risk factors (i.e., antithrombosis agents, antihypertensive drugs, statins, and oral antidiabetes therapy) which may affect the measures of cortical excitability and conductivity. The same holds for psychotropic drugs often taken for the treatment of anxiety and depressive disorders (i.e., benzodiazepines, antidepressants, antipsychotics, and mood stabilizers) [44, 45, 57]. Therefore, the study design should consider patients under medicaments which, to the best of current knowledge, have no or limited influence at the level of TMS.

(h) Finally, a usual limitation in TMS research is the threshold of sensitivity and specificity of the TMS measures when used as a diagnostic tool. In order 
to adequately define sensitivity and specificity, the individual measures in all the patients and controls, not only the mean values, would be required. Moreover, to estimate the number of false positives, the whole subject population needs to be followed up independently to assess if they show clinical deterioration [34]. Up to now, very few studies fulfill these requirements, and the application of TMS in dementia needs future studies with methodological improvement and high degree of standardization. Nevertheless, in view of the exponential growth of new and sometimes not concordant data, this review aimed to consolidate, wherever possible, the available knowledge on TMS and VCI.

\section{Conclusion, Translational Value, and Future Perspectives}

To date, the development of dementia cannot be accurately predicted by conventional investigations [115]. However, unlike degenerative dementias, $\mathrm{VaD}$ can be prevented, at least in part, in most of the patients through a careful prevention and a close monitoring of vascular risk factors. Preventing VCI means to prevent vascular accidents and future dementia, to reduce mortality, disability, and institutionalization rates, to maintain an acceptable functional status in the elderly, and, ultimately, to save clinical-social costs [116].

The effect of conventional treatment on VCI and $\mathrm{VaD}$ is not well established. Moreover, the heterogeneous construct of VCI constitutes a challenge in the selection of appropriate outcome measures in clinical trials of pharmacological interventions. Therefore, the discovery of new early targets to prevent or treat $\mathrm{VaD}$ is desirable. In this context, despite the fact that patient cohorts and methodologies are not always homogeneous between studies, much of the literature agrees on the utility of TMS in dementias [34]. Although a single measure is not sufficient to define a diagnosis, all together the parameters of interest are footprints of specific pathophysiological processes that affect motor and nonmotor areas in various forms of dementia. Taken together, the available TMS evidences point out the possibility to identify a specific profile of cortical excitability each related to a VCI subtype, and in particular to predict conversion of the so-called "brain at risk" for VaD into an overt dementia. This will be of pivotal importance when designing trials of diseasemodifying drugs and innovative nonpharmacological approaches based on brain stimulation. Conversely, in patients with overt dementia, TMS can be exploited to select and evaluate the responders to specific drugs and might also become a rehabilitative tool in the attempt to restore impaired brain plasticity.

Although it is not possible at the moment to determine whether findings from the studies here reviewed are reflected in decision-making in the care of patients, TMS might prompt a design of an instrument for screening of population at risk, studying drug-induced changes in the electrical properties of the human cortex, probing models of brain connectivity, and testing neuromodulatory therapeutic tools for cognitive rehabilitation. When patients at risk are identified, a more careful prevention and control of vascular risk factors should be considered. Further longitudinal studies combining TMS with other neurophysiological techniques (including high-density EEG and event-related potentials) and advanced structural and functional imaging data (such as functional MRI and diffusion tensor imaging), as well as serum and CSF biological markers will clarify the impact of vascular cognitive, mood, and behavioral deficits on cortical excitability and synaptic plasticity.

\section{Competing Interests}

The authors declare that there is no conflict of interest regarding the publication of this paper.

\section{References}

[1] G. C. Román, P. Sachdev, D. R. Royall et al., "Vascular cognitive disorder: a new diagnostic category updating vascular cognitive impairment and vascular dementia," Journal of the Neurological Sciences, vol. 226, no. 1-2, pp. 81-87, 2004.

[2] P. Moorhouse and K. Rockwood, "Vascular cognitive impairment: current concepts and clinical developments," The Lancet Neurology, vol. 7, no. 3, pp. 246-255, 2008.

[3] C. Ballard, E. Rowan, S. Stephens, R. Kalaria, and R. A. Kenny, "Prospective follow-up study between 3 and 15 months after stroke: improvements and decline in cognitive function among dementia-free stroke survivors $>75$ years of age," Stroke, vol. 34, no. 10, pp. 2440-2444, 2003.

[4] R. O. Akinyemi, L. Allan, M. O. Owolabi et al., "Profile and determinants of vascular cognitive impairment in African stroke survivors: the CogFAST Nigeria Study," Journal of the Neurological Sciences, vol. 346, no. 1-2, pp. 241-249, 2014.

[5] R. N. Kalaria, R. Akinyemi, and M. Ihara, "Stroke injury, cognitive impairment and vascular dementia," Biochimica et Biophysica Acta, vol. 1862, no. 5, pp. 915-925, 2016.

[6] B. P. Vasquez and K. K. Zakzanis, "The neuropsychological profile of vascular cognitive impairment not demented: a meta-analysis," Journal of Neuropsychology, vol. 9, no. 1, pp. 109-136, 2015.

[7] M. Gupta, A. Dasgupta, G. A. Khwaja, D. Chowdhury, Y. Patidar, and A. Batra, "Behavioural and psychological symptoms in poststroke vascular cognitive impairment," Behavioural Neurology, Article ID 430128, p. 5, 2014.

[8] R. N. Kalaria and C. Ballard, "Stroke and cognition," Current Atherosclerosis Reports, vol. 3, no. 4, pp. 334-339, 2001.

[9] R. N. Kalaria, "Cerebrovascular disease and mechanisms of cognitive impairment: evidence from clinicopathological studies in humans," Stroke, vol. 43, no. 9, pp. 2526-2534, 2012.

[10] J. T. O’Brien, T. Erkinjuntti, B. Reisberg et al., "Vascular cognitive impairment," Lancet Neurology, vol. 2, no. 2, pp. 89-98, 2003.

[11] D. Inzitari, G. Pracucci, A. Poggesi et al., "Changes in white matter as determinant of global functional decline in older independent outpatients: three year follow-up of LADIS (leukoaraiosis and disability) study cohort," The British Medical Journal, vol. 339, p. b2477, 2009. 
[12] D. C. Steffens, M. J. Helms, K. R. Krishnan, and G. L. Burke, "Cerebrovascular disease and depression symptoms in the cardiovascular health study," Stroke, vol. 30, no. 10, pp. 2159-2166, 1999.

[13] J. C. de Groot, F. de Leeuw, M. Oudkerk, A. Hofman, J. Jolles, and M. M. B. Breteler, "Cerebral white matter lesions and depressive symptoms in elderly adults," Archives of General Psychiatry, vol. 57, no. 11, pp. 1071-1076, 2000.

[14] A. Teodorczuk, J. T. O’Brien, M. J. Firbank et al., "White matter changes and late-life depressive symptoms," British Journal of Psychiatry, vol. 191, no. 3, pp. 212-217, 2007.

[15] R. M. Bonelli and J. L. Cummings, "Frontal-subcortical circuitry and behaviour," Dialogues in Clinical Neuroscience, vol. 9, no. 2, pp. 141-151, 2007.

[16] R. O. Akinyemi, M. Firbank, G. I. Ogbole et al., "Medial temporal lobe atrophy, white matter hyperintensities and cognitive impairment among Nigerian African stroke survivors," BMC Research Notes, vol. 8, no. 1, p. 625, 2015.

[17] A. Chen, R. O. Akinyemi, Y. Hase et al., "Frontal white matter hyperintensities, clasmatodendrosis and gliovascular abnormalities in ageing and post-stroke dementia," Brain, vol. 139, part 1, pp. 242-258, 2016.

[18] A. Ojagbemi, R. Akinyemi, and O. Baiyewu, "Cognitive dysfunction and functional limitations are associated with major depression in stroke survivors attending rehabilitation in Nigeria," Neuro Rehabilitation, vol. 34, no. 3, pp. 455-461, 2014.

[19] N. D. Prins, E. J. van Dijk, T. den Heijer et al., "Cerebral small-vessel disease and decline in information processing speed, executive function and memory," Brain, vol. 128, part 9, pp. 2034-2041, 2005.

[20] G. S. Alexopoulos, B. S. Meyers, R. C. Young, S. Campbell, D. Silbersweig, and M. Charlson, "Vascular depression" hypothesis," Archives of General Psychiatry, vol. 54, no. 10, pp. 915-922, 1997.

[21] J. O’Brien, D. Ames, E. Chiu, I. Schweitzer, P. Desmond, and B. Tress, "Severe deep white matter lesions and outcome in elderly patients with major depressive disorder: follow up study," BMJ, vol. 317, no. 7164, pp. 982-984, 1998.

[22] M. Liotti and H. S. Mayberg, "The role of functional neuroimaging in the neuropsychology of depression," Journal of Clinical and Experimental Neuropsychology, vol. 23, no. 1, pp. 121-136, 2001.

[23] D. C. Steffens, "Establishing diagnostic criteria for vascular depression," Journal of Neurological Sciences, vol. 226, no. 1-2, pp. 59-62, 2004.

[24] L. Pantoni, F. Fierini, A. Poggesi, and LADIS Study Group, "Impact of cerebral white matter changes on functionality in older adults: an overview of the LADIS Study results and future directions," Geriatrics \& Gerontology International, vol. 15, Supplement 1, pp. 10-16, 2015.

[25] C. F. Murphy, F. M. Gunning-Dixon, M. J. Hoptman et al., "White-matter integrity predicts stroop performance in patients with geriatric depression," Biological Psychiatry, vol. 61, no. 8, pp. 1007-1010, 2007.

[26] G. S. Alexopoulos, D. N. Kiosses, M. Heo, C. F. Murphy, B. Shanmugham, and F. Gunning-Dixon, "Executive dysfunction and the course of geriatric depression," Biological Psychiatry, vol. 58, no. 3, pp. 204-210, 2005.

[27] A. J. Thomas, J. T. O’Brien, S. Davis et al., "Ischemic basis for deep white matter hyperintensities in major depression: a neuropathological study," Archives of General Psychiatry, vol. 59, no. 9, pp. 785-792, 2002.

[28] A. Teodorczuk, M. J. Firbank, L. Pantoni et al., "Relationship between baseline white-matter changes and development of late-life depressive symptoms: 3-year results from the LADIS Study," Psychological Medicine, vol. 40, no. 4, pp. 603-610, 2010.

[29] R. Bella, G. Pennisi, M. Cantone et al., "Clinical presentation and outcome of geriatric depression in subcortical ischemic vascular disease," Gerontology, vol. 56, no. 3, pp. 298-302, 2010.

[30] G. Pennisi, R. Bella, and G. Lanza, "Motor cortex plasticity in subcortical ischemic vascular dementia: what can TMS say?" Clinical Neurophysiology, vol. 126, no. 5, pp. 851-852, 2015.

[31] R. N. Kalaria, "Risk factors and neurodegenerative mechanisms in stroke related dementia," Panminerva Medica, vol. 54, no. 3, pp. 139-148, 2012.

[32] R. O. Akinyemi, E. B. Mukaetova-Ladinska, J. Attems, M. Ihara, and R. N. Kalaria, "Vascular risk factors and neurodegeneration in ageing related dementias: Alzheimer's disease and vascular dementia," Current Alzheimer Research, vol. 10, no. 6, pp. 642-653, 2013.

[33] R. N. Kalaria, "Neuropathological diagnosis of vascular cognitive impairment and vascular dementia with implications for Alzheimer's disease," Acta Neuropathologica, vol. 131, no. 5, pp. 659-685, 2016.

[34] M. Cantone, G. Di Pino, F. Capone et al., "The contribution of transcranial magnetic stimulation in the diagnosis and in the management of dementia," Clinical Neurophysiology, vol. 125, no. 8, pp. 1509-1532, 2014.

[35] R. Chen, D. Cros, A. Curra et al., "The clinical diagnostic utility of transcranial magnetic stimulation: report of an IFCN committee," Clinical Neurophysiology, vol. 119, no. 3, pp. 504-532, 2008.

[36] M. Kobayashi and A. Pascual-Leone, "Transcranial magnetic stimulation in neurology," The Lancet Neurology, vol. 2, no. 3, pp. 145-156, 2003.

[37] G. Lanza, B. Lanuzza, D. Aricò et al., "Direct comparison of cortical excitability to transcranial magnetic stimulation in obstructive sleep apnea syndrome and restless legs syndrome," Sleep Medicine, vol. 16, no. 1, pp. 138-142, 2015.

[38] G. Pennisi, G. Lanza, S. Giuffrida et al., "Excitability of the motor cortex in de novo patients with celiac disease," PLoS One, vol. 9, no. 7, p. e102790, 2014.

[39] R. Bella, G. Lanza, M. Cantone et al., "Effect of a gluten-free diet on cortical excitability in adults with celiac disease," PLoS One, vol. 10, no. 6, p. e0129218, 2015.

[40] P. M. Rossini, S. Rossi, C. Babiloni, and J. Polich, "Clinical neurophysiology of aging brain: from normal aging to neurodegeneration," Progress in Neurobiology, vol. 83, no. 6, pp. 375-400, 2007.

[41] S. Groppa, "Multifocal TMS for temporo-spatial description of cortico-cortical connectivity patterns," Clinical Neurophysiology, vol. 127, no. 2, pp. 1005-1006, 2016.

[42] F. Farzan, M. Vernet, M. M. Shafi, A. Rotenberg, Z. J. Daskalakis, and A. Pascual-Leone, "Characterizing and modulating brain circuitry through transcranial magnetic stimulation combined with electroencephalography," Frontiers in Neural Circuits, vol. 10, p. 73, 2016.

[43] V. K. Kimiskidis, “Transcranial magnetic stimulation (TMS) coupled with electroencephalography (EEG): biomarker of 
the future," The Revue Neurologique, vol. 172, no. 2, pp. 123126, 2016.

[44] W. Paulus, J. Classen, L. G. Cohen et al., "State of the art: pharmacologic effects on cortical excitability measures tested by transcranial magnetic stimulation," Brain Stimulation, vol. 1, no. 3, pp. 151-163, 2008.

[45] U. Ziemann, J. Reis, P. Schwenkreis et al., "TMS and drugs revisited 2014," Clinical Neurophysiology, vol. 126, no. 10, pp. 1847-1868, 2015.

[46] V. Di Lazzaro, A. Oliviero, P. Mazzone et al., "Comparison of descending volleys evoked by monophasic and biphasic magnetic stimulation of the motor cortex in conscious humans," Experimental Brain Research, vol. 141, no. 1, pp. 121-127, 2001.

[47] P. M. Rossini, D. Burke, R. Chen et al., "Non-invasive electri$\mathrm{cal}$ and magnetic stimulation of the brain, spinal cord, roots and peripheral nerves: basic principles and procedures for routine clinical and research application. An updated report from an I.F.C.N. Committee," Clinical Neurophysiology, vol. 126, no. 6, pp. 1071-1107, 2015.

[48] P. M. Rossini and S. Rossi, "Transcranial magnetic stimulation: diagnostic, therapeutic, and research potential," Neurology, vol. 68, no. 7, pp. 484-488, 2007.

[49] R. Chen, A. M. Lozano, and P. Ashby, "Mechanism of the silent period following transcranial magnetic stimulation. Evidence from epidural recordings," Experimental Brain Research, vol. 128, no. 4, pp. 539-542, 1999.

[50] R. Cantello, M. Gianelli, C. Civardi, and R. Mutani, "Magnetic brain stimulation: the silent period after the motor evoked potential," Neurology, vol. 42, no. 10, pp. 1951-1959, 1992.

[51] K. J. Werhahn, E. Kunesch, S. Noachtar, R. Benecke, and J. Classen, "Differential effects on motorcortical inhibition induced by blockade of GABA uptake in humans," Journal of Physiology, vol. 517, part 2, pp. 591-597, 1999.

[52] H. R. Siebner, J. Dressnandt, C. Auer, and B. Conrad, "Continuous intrathecal baclofen infusions induced a marked increase of the transcranially evoked silent period in a patient with generalized dystonia," Muscle \& Nerve, vol. 21, no. 9, pp. 1209-1212, 1998.

[53] T. Kujirai, M. D. Caramia, J. C. Rothwell et al., "Corticocortical inhibition in human motor cortex," The Journal of Physiology, vol. 471, pp. 501-519, 1993.

[54] U. Ziemann, J. C. Rothwell, and M. C. Ridding, "Interaction between intracortical inhibition and facilitation in human motor cortex," Journal of Physiology, vol. 496, part 3, pp. 873-881, 1996.

[55] V. Di Lazzaro, F. Pilato, M. Dileone et al., "GABAA receptor subtype specific enhancement of inhibition in human motor cortex," Journal of Physiology, vol. 575, part 3, pp. 721-726, 2006.

[56] V. Di Lazzaro, F. Pilato, A. Oliviero et al., "Origin of facilitation of motor-evoked potentials after paired magnetic stimulation: direct recording of epidural activity in conscious humans," Journal of Neurophysiology, vol. 96, no. 4, pp. 1765-1771, 2006.

[57] U. Ziemann, “TMS and drugs," Clinical Neurophysiology, vol. 115, no. 8, pp. 1717-1729, 2004.

[58] H. Tokimura, V. Di Lazzaro, Y. Tokimura et al., "Short latency inhibition of human hand motor cortex by somatosensory input from the hand," Journal of Physiology, vol. 523, part 2, pp. 503-513, 2000.
[59] V. Di Lazzaro, A. Oliviero, P. Profice et al., "Muscarinic receptor blockade has differential effects on the excitability of intracortical circuits in the human motor cortex," Experimental Brain Research, vol. 135, no. 4, pp. 455-461, 2000.

[60] V. Di Lazzaro, A. Oliviero, F. Pilato et al., "Neurophysiological predictors of long term response to AChE inhibitors in AD patients," Journal of Neurology, Neurosurgery and Psychiatry, vol. 76, no. 8, pp. 1064-1069, 2005.

[61] A. Sailer, G. F. Molnar, G. Paradiso, C. A. Gunraj, A. E. Lang, and R. Chen, "Short and long latency afferent inhibition in Parkinson's disease," Brain, vol. 126, part 8, pp. 1883-1894, 2003.

[62] A. Martorana, F. Mori, Z. Esposito et al., "Dopamine modulates cholinergic cortical excitability in Alzheimer's disease patients," Neuropsychopharmacology, vol. 34, no. 10, pp. 2323-2328, 2009.

[63] M. D. Caramia, A. Scalise, R. Gordon, H. J. Michalewski, and A. Starr, "Delayed facilitation of motor cortical excitability following repetitive finger movements," Clinical Neurophysiology, vol. 111, no. 9, pp. 1654-1660, 2000.

[64] R. Chen, J. Classen, C. Gerloff et al., "Depression of motor cortex excitability by low-frequency transcranial magnetic stimulation," Neurology, vol. 48, no. 5, pp. 1398-1403, 1997.

[65] A. Berardelli, M. Inghilleri, J. C. Rothwell et al., "Facilitation of muscle evoked responses after repetitive cortical stimulation in man," Experimental Brain Research, vol. 122, no. 1, pp. 79-84, 1998.

[66] C. Spampinato, E. Aguglia, C. Concerto et al., "Transcranial magnetic stimulation in the assessment of motor cortex excitability and treatment of drug-resistant major depression," IEEE Transactions on Neural Systems \& Rehabilitation Engineering, vol. 21, no. 3, pp. 391-403, 2013.

[67] C. Concerto, G. Lanza, M. Cantone et al., "Repetitive transcranial magnetic stimulation in patients with drug-resistant major depression: a six-month clinical follow-up study," International Journal of Psychiatry in Clinical Practice, vol. 19, no. 4, pp. 252-258, 2015.

[68] S. Bashir, I. Mizrahi, K. Weaver, F. Fregni, and A. PascualLeone, "Assessment and modulation of neural plasticity in rehabilitation with transcranial magnetic stimulation," $P M$ \& $R$, vol. 2, no. 12, Supplement 2, pp. S253-S268, 2010.

[69] L. G. Cohen, U. Ziemann, R. Chen et al., "Studies of neuroplasticity with transcranial magnetic stimulation," Journal of Clinical Neurophysiology, vol. 15, no. 4, pp. 305-324, 1998.

[70] R. J. Anderson, K. E. Hoy, Z. J. Daskalakis, and P. B. Fitzgerald, "Repetitive transcranial magnetic stimulation for treatment resistant depression: re-establishing connections," Clinical Neurophysiology, vol. 127, no. 11, pp. 3394-3405, 2016.

[71] A. Floel and L. G. Cohen, "Contribution of noninvasive cortical stimulation to the study of memory functions," Brain Research Reviews, vol. 53, no. 2, pp. 250-259, 2007.

[72] J. M. Hoogendam, G. M. Ramakers, and V. Di Lazzaro, "Physiology of repetitive transcranial magnetic stimulation of the human brain," Brain Stimulation, vol. 3, no. 2, pp. 95-118, 2010.

[73] K. Stefan, E. Kunesch, R. Benecke, L. G. Cohen, and J. Classen, "Mechanisms of enhancement of human motor cortex excitability induced by interventional paired associative stimulation," Journal of Physiology, vol. 543, part 2, pp. 699-708, 2002. 
[74] I. Delvendahl, N. H. Jung, N. G. Kuhnke, U. Ziemann, and V. Mall, "Plasticity of motor threshold and motor-evoked potential amplitude-a model of intrinsic and synaptic plasticity in human motor cortex?" Brain Stimulation, vol. 5, no. 4, pp. 586-593, 2012.

[75] G. Lanza, M. Cantone, B. Lanuzza et al., "Distinctive patterns of cortical excitability to transcranial magnetic stimulation in obstructive sleep apnea syndrome, restless legs syndrome, insomnia, and sleep deprivation," Sleep Medicine Reviews, vol. 19, pp. 39-50, 2015.

[76] C. Concerto, G. Lanza, M. Cantone et al., "Different patterns of cortical excitability in major depression and vascular depression: a transcranial magnetic stimulation study," BMC Psychiatry, vol. 13, no. 1, p. 300, 2013.

[77] G. Pennisi, R. Ferri, G. Lanza et al., "Transcranial magnetic stimulation in Alzheimer's disease: a neurophysiological marker of cortical hyperexcitability," Journal of Neural Transmission, vol. 118, no. 4, pp. 587-598, 2011.

[78] G. Pennisi, R. Ferri, M. Cantone et al., "A review of transcranial magnetic stimulation in vascular dementia," Dementia and Geriatric Cognitive Disorders, vol. 31, no. 1, pp. 71-80, 2011.

[79] F. Ferreri and P. M. Rossini, "TMS and TMS-EEG techniques in the study of the excitability, connectivity, and plasticity of the human motor cortex," Reviews in the Neurosciences, vol. 24, no. 4, pp. 431-442, 2013.

[80] G. Alagona, R. Ferri, G. Pennisi et al., "Motor cortex excitability in Alzheimer's disease and in subcortical ischemic vascular dementia," Neuroscience Letters, vol. 362, no. 2, pp. 9598, 2004.

[81] V. Di Lazzaro, F. Pilato, M. Di Leone et al., "In vivo functional evaluation of central cholinergic circuits in vascular dementia," Clinical Neurophysiology, vol. 119, no. 11, pp. 24942500, 2008.

[82] R. Nardone, J. Bergmann, F. Tezzon, G. Ladurner, and S. Golaszewski, "Cholinergic dysfunction in subcortical ischaemic vascular dementia: a transcranial magnetic stimulation study," Journal of Neural Transmission, vol. 115, no. 5, pp. 737-743, 2008.

[83] F. Manganelli, M. Ragno, G. Cacchiò et al., "Motor cortex cholinergic dysfunction in CADASIL: a transcranial magnetic demonstration," Clinical Neurophysiology, vol. 119, no. 2, pp. 351-355, 2008.

[84] G. Pennisi, R. Ferri, G. Alagona et al., "Motor cortex hyperexcitability in subcortical ischemic vascular dementia," Archives of Gerontology and Geriatrics, vol. 53, no. 2, pp. e111-e113, 2011.

[85] R. Nardone, P. De Blasi, M. Seidl et al., "Cognitive function and cholinergic transmission in patients with subcortical vascular dementia and microbleeds: a TMS study," Journal of Neural Transmission, vol. 118, no. 9, pp. 1349-1358, 2011.

[86] R. Bella, R. Ferri, M. Cantone et al., "Motor cortex excitability in vascular depression," International Journal of Psychophysiology, vol. 82, no. 3, pp. 248-253, 2011.

[87] R. Bella, R. Ferri, M. Pennisi et al., "Enhanced motor cortex facilitation in patients with vascular cognitive impairmentno dementia," Neuroscience Letters, vol. 503, no. 3, pp. 171175, 2011.

[88] R. Bella, R. Ferri, G. Lanza et al., "TMS follow-up study in patients with vascular cognitive impairment-no dementia," Neuroscience Letters, vol. 534, pp. 155-159, 2013.
[89] G. Lanza, R. Bella, S. Giuffrida et al., "Preserved transcallosal inhibition to transcranial magnetic stimulation in nondemented elderly patients with leukoaraiosis," BioMed Research International, vol. 2013, Article ID 351680, p. 5, 2013.

[90] J. List, T. Duning, J. Kürten, M. Deppe, E. Wilbers, and A. Flöel, "Cortical plasticity is preserved in nondemented older individuals with severe ischemic small vessel disease," Human Brain Mapping, vol. 34, no. 6, pp. 1464-1476, 2013.

[91] F. J. Palomar, A. Suárez, E. Franco, F. Carrillo, E. Gil-Néciga, and P. Mir, "Abnormal sensorimotor plasticity in CADASIL correlates with neuropsychological impairment," Journal of Neurology, Neurosurgery, and Psychiatry, vol. 84, no. 3, pp. 329-336, 2013.

[92] R. Nardone, Y. Höller, A. Thomschewski et al., "Dopamine differently modulates central cholinergic circuits in patients with Alzheimer disease and CADASIL," Journal of Neural Transmission, vol. 121, no. 10, pp. 1313-1320, 2014.

[93] J. List, S. Hertel-Zens, J. C. Kübke, A. Lesemann, S. J. Schreiber, and A. Flöel, "Cortical reorganization due to impaired cerebral autoregulation in individuals with occlusive processes of the internal carotid artery," Brain Stimulation, vol. 7, no. 3, pp. 381-387, 2014.

[94] A. Guerra, S. Petrichella, L. Vollero et al., "Neurophysiological features of motor cortex excitability and plasticity in subcortical ischemic vascular dementia: a TMS mapping study," Clinical Neurophysiology, vol. 126, no. 5, pp. 906-913, 2015.

[95] R. Bella, M. Cantone, G. Lanza et al., "Cholinergic circuitry functioning in patients with vascular cognitive impairment-no dementia," Brain Stimulation, vol. 9, no. 2, pp. 225-233, 2016.

[96] M. Pennisi, G. Lanza, M. Cantone et al., "Correlation between motor cortex excitability changes and cognitive impairment in vascular depression: pathophysiological insights from a longitudinal TMS study," Neural Plasticity, vol. 2016, Article ID 8154969, p. 10, 2016.

[97] T. Erkinjuntti, D. Inzitari, L. Pantoni et al., "Research criteria for subcortical vascular dementia in clinical trials," The Journal of Neural Transmission. Supplementa, vol. 59, pp. 23-30, 2000.

[98] F. Ferreri, F. Pauri, P. Pasqualetti, R. Fini, G. Dal Forno, and P. M. Rossini, "Motor cortex excitability in Alzheimer's disease: a transcranial magnetic stimulation study," Annals of Neurology, vol. 53, no. 1, pp. 102-108, 2003.

[99] R. Nardone, J. Bergmann, M. Christova et al., "Short latency afferent inhibition differs among the subtypes of mild cognitive impairment," Journal of Neural Transmission, vol. 119, no. 4, pp. 463-471, 2012.

[100] R. Nardone, S. Golaszewski, G. Ladurner, F. Tezzon, and E. Trinka, "A review of transcranial magnetic stimulation in the in vivo functional evaluation of central cholinergic circuits in dementia," Dementia and Geriatric Cognitive Disorders, vol. 32, no. 1, pp. 18-25, 2011.

[101] R. H. Swartz, D. J. Sahlas, and S. E. Black, "Strategic involvement of cholinergic pathways and executive dysfunction: does location of white matter signal hyperintensities matter?" Journal of Stroke and Cerebrovascular Diseases, vol. 12, no. 1, pp. 29-36, 2003.

[102] M. O’Sullivan, J. M. Jarosz, R. J. Martin, N. Deasy, J. F. Powell, and H. S. Markus, "MRI hyperintensities of the temporal lobe and external capsule in patients with CADASIL," Neurology, vol. 56, no. 5, pp. 628-634, 2001. 
[103] J. List, T. Duning, M. Meinzer et al., "Enhanced rapid-onset cortical plasticity in CADASIL as a possible mechanism of preserved cognition," Cerebral Cortex, vol. 21, no. 12, pp. 2774-2787, 2011.

[104] G. J. Elder and J. P. Taylor, “Transcranial magnetic stimulation and transcranial direct current stimulation: treatments for cognitive and neuropsychiatric symptoms in the neurodegenerative dementias?" Alzheimer's Research \& Therapy, vol. 6, no. 9, p. 74, 2015.

[105] S. Rossi and P. M. Rossini, "TMS in cognitive plasticity and the potential for rehabilitation," Trends in Cognitive Sciences, vol. 8, no. 6, pp. 273-279, 2004.

[106] C. Miniussi and P. M. Rossini, "Transcranial magnetic stimulation in cognitive rehabilitation," Neuropsychological Rehabilitation, vol. 21, no. 5, pp. 579-601, 2011.

[107] I. Rektorova, S. Megova, M. Bares, and I. Rektor, "Cognitive functioning after repetitive transcranial magnetic stimulation in patients with cerebrovascular disease without dementia: a pilot study of seven patients," The Journal of the Neurological Sciences, vol. 229-230, pp. 157-161, 2005.

[108] A. R. Brunoni, I. M. Benseñor, and T. C. Alves, "Therapeutic interventions for vascular depression: a systematic review," Revista Brasileira de Psiquiatria, vol. 33, no. 4, pp. 400-409, 2011.

[109] A. McIntyre, S. Thompson, A. Burhan, S. Mehta, and R. Teasell, "Repetitive transcranial magnetic stimulation for depression due to cerebrovascular disease: a systematic review," Journal of Stroke Cerebrovascular Diseases, vol. 25, no. 12, pp. 2792-2800, 2016.

[110] H. Yang, O. Shi, Y. Jin et al., "Functional protection of learning and memory abilities in rats with vascular dementia," Restorative Neurology and Neuroscience, vol. 32, no. 5, pp. 689-700, 2014.

[111] F. Wang, G. M. Chang, Q. Yu, and X. Geng, "The neuroprotection of repetitive transcranial magnetic stimulation pretreatment in vascular dementia rats," Journal of Molecular Neuroscience, vol. 56, no. 1, pp. 198-204, 2015.

[112] B. Fritsch, J. Reis, K. Martinowich et al., "Direct current stimulation promotes BDNF-dependent synaptic plasticity: potential implications for motor learning," Neuron, vol. 66, no. 2, pp. 198-204, 2010.

[113] T. Yukimasa, R. Yoshimura, A. Tamagawa et al., "High-frequency repetitive transcranial magnetic stimulation improves refractory depression by influencing catecholamine and brain-derived neurotrophic factors," Pharmacopsychiatry, vol. 39, no. 2, pp. 52-59, 2006.

[114] H. Y. Yang, Y. Liu, J. C. Xie, N. N. Liu, and X. Tian, "Effects of repetitive transcranial magnetic stimulation on synaptic plasticity and apoptosis in vascular dementia rats," Behavioural Brain Research, vol. 281, pp. 149-155, 2015.

[115] A. M. Hakim, "Vascular disease: the tsunami of health care," Stroke, vol. 38, no. 12, pp. 3296-3301, 2007.

[116] M. O. Owolabi, S. Akarolo-Anthony, R. Akinyemi et al., "The burden of stroke in Africa: a glance at the present and a glimpse into the future," Cardiovascular Journal of Africa, vol. 26, no. 2, Supplement 1, pp. S27-S38, 2015. 


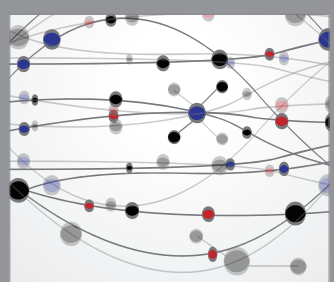

The Scientific World Journal
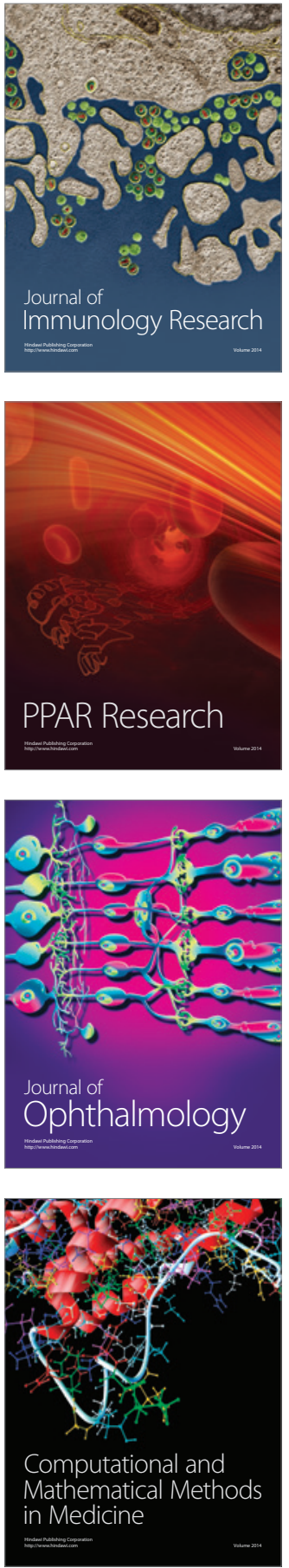

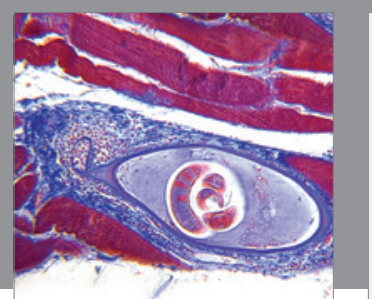

Gastroenterology Research and Practice
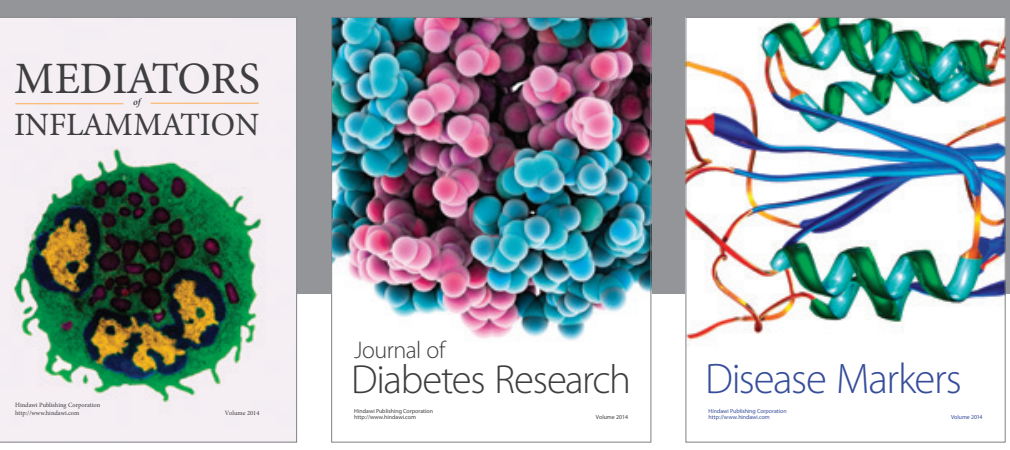

Disease Markers

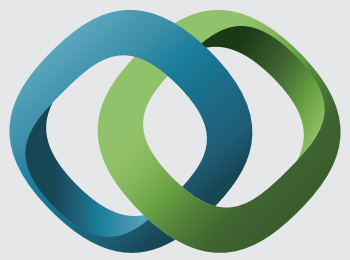

\section{Hindawi}

Submit your manuscripts at

https://www.hindawi.com
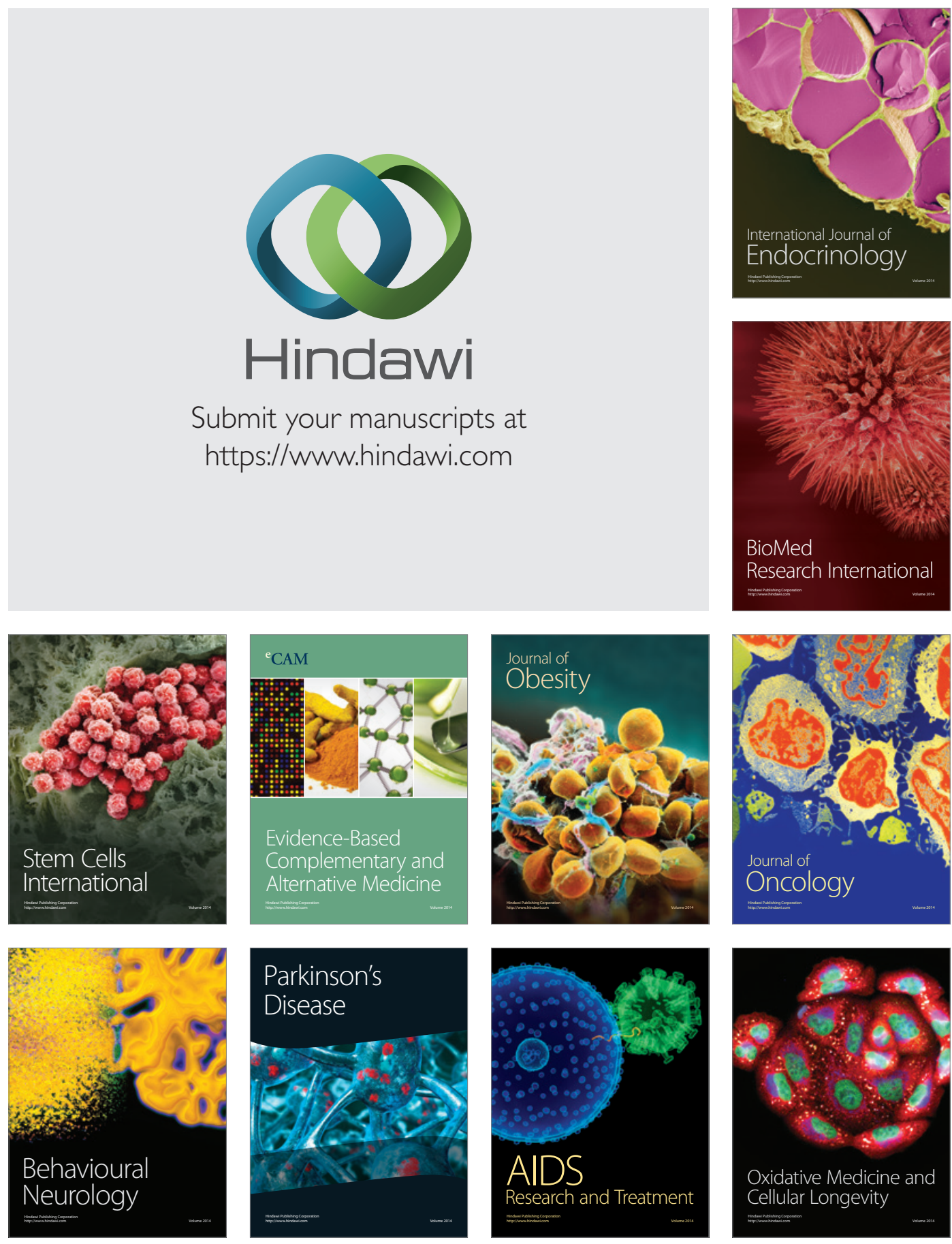